\title{
Role of Lymphocytes CD4/CD8 Ratio and Immunoglobulin G Cytomegalovirus as Potential Markers for Systemic Lupus Erythematosus Patients with Periodontal Disease
}

\author{
Kusworini Handono ${ }^{1}$ Nanda Rachmad Putra Gofur ${ }^{2}$ Nurdiana Nurdiana ${ }^{3}$ Handono Kalim ${ }^{4}$ \\ Cesarius Singgih Wahono ${ }^{4}$ Sri Poeranto ${ }^{5}$ Wisnu Barlianto ${ }^{6}$ \\ ${ }^{1}$ Department of Clinical Pathology, Faculty of Medicine, \\ Universitas Brawijaya, Malang, Indonesia \\ 2Biomedical Science, Postgraduate Program, Faculty of Medicine, \\ Universitas Brawijaya, Malang, Indonesia \\ ${ }^{3}$ Department of Pharmacology, Faculty of Medicine, \\ Universitas Brawijaya, Malang, Indonesia \\ ${ }^{4}$ Department of Internal Medicine, Faculty of Medicine, \\ Universitas Brawijaya, Malang, Indonesia \\ ${ }^{5}$ Department of Parasitology, Faculty of Medicine, Universitas \\ Brawijaya, Malang, Indonesia \\ 6 Department of Pediatric, Faculty of Medicine, \\ Universitas Brawijaya, Malang, Indonesia \\ Eur J Dent:2020;14:544-550

\begin{abstract}
Address for correspondence Kusworini Handono, Department of Pathology Clinic, Faculty of Medicine, Universitas Brawijaya, Veteran 45 Malang, Indonesia (e-mail: dr.kusworini@gmail.com).
\end{abstract}

\begin{abstract}
Objectives The aim of the study was to analyze the correlation between periodontitis severity in systemic lupus erythematosus (SLE) with CD4/CD8 lymphocytes ratio and cytomegalovirus gamma immunoglobulin (IgG CMV) level.

Materials and Methods This is a descriptive study using a cross-sectional approach that included 93 subjects who were diagnosed with SLE in Rheumatology Department, Saiful Anwar Hospital, during 2017 to 2019. Periodontitis severity was assessed by periodontal Index (PI). CD4/CD8 lymphocyte ratio was determined using flow cytometry and IgG CMV levels using enzyme-linked immunosorbent assay.

Statistical Analysis The differences among the three groups were analyzed using analysis of variance. Correlation among the groups was calculated using Spearman/ Pearson correlation coefficient test, while regression analysis was done using Statistical Package for the Social Sciences.

Results The mean of periodontitis severity and standard deviation in SLE was

Keywords

- periodontitis

- systemic lupus erythematous

- CD4/CD8 ratio

$-\lg G \mathrm{CMV}$ $2.66 \pm 1.02$. There were negative correlation between CD4/CD8 lymphocyte ratio with periodontal index $(r=-0.971)$ and positive correlation between IgG CMV level with periodontal index $(r=0.977)$.

Conclusions Inverted CD4/CD8 ratio and IgG CMV were found associated with periodontitis severity in SLE patient. Further research was recomended that CD4/CD8 lymphocytes ratio and IgG CMV can be used as a potensial marker of periodontitis severity in SLE patients.
\end{abstract}

DOI https://doi.org/

$10.1055 / \mathrm{s}-0040-1715788$

ISSN 1305-7456.
License terms

() (1) $\ominus \circledast$ 


\section{Introduction}

Periodontal diseases are bacterial infections affecting the periodontium, beginning from gingival tissue. Furthermore, infection could induce chronic inflammation resulting in tooth support tissue destruction. Chronic inflammation could worsen due to systemic conditions that follow. Activation of worsened host immune response may be associated with autoimmune conditions, especially in case of systemic lupus erythematosus (SLE). Hyperactive immune response system in SLE could cause peripheral tissue damages, such as mucosal tissue and periodontal tissue., ${ }^{1,2}$

Mucosal tissue damages resulting in mucosal ulcer, specifically, could be a sign for early manifestations in SLE. Moreover, multiple oral ulcer findings were used for diagnosis in Systemic Lupus International Collaborating Clinics criteria for SLE patients. Research also found other manifestations in SLE such as xerostomia and decay (80-95\%). On the other hand, periodontitis was found in 50 to $75 \%$ of SLE cases. ${ }^{3}$ Worldwide, periodontitis in SLE is higher compared with healthy subjects in several studies. ${ }^{4,5}$ Preliminary study in Indonesia concludes that periodontitis is associated with SLE. Early periodontitis is characterized by inflammation of gums, without attachment loss. Clinical signs of advanced periodontitis are attachment loss, inflammation of the gingiva, and bone breakdown, which are found to be higher (88.53\%) in SLE patients than control. ${ }^{6,7}$

Periodontitis starts as gingival inflammation at the gingival margin and causes irreversible damage to the periodontium. Inflammation severity may vary among individuals based on the degree of bacterial infection, and dysregulation of host inflammatory response may substantially contribute to its extent. In autoimmune conditions, especially SLE, periodontitis pathway is influenced by systemic conditions from the host. ${ }^{8}$ Hence, host condition could affect periodontitis especially due to immune responses. Patients with autoimmune disease affect system immune responses resulting in change in innate and adaptive immune, chemokine, metalloproteinase, and cytokines production in blood. A previous study showed that periodontitis can be compounded by autoimmune conditions. ${ }^{9}$ One of the autoimmune diseases that is believed to be a high-risk factor for periodontitis is SLE. ${ }^{8,10}$

SLE incidence occurs more often in a young female. The incidence of SLE has continued to increase almost three times in the past 40 years, mainly due to better diagnosis. SLE is also found more commonly in urban than in rural areas. SLE manifests in multiple organs, could attack tissues and cells, and is known as a disease with thousand faces. ${ }^{11}$ Hyperactive immune responses in SLE could induce an inverted $\mathrm{T}$ lymphocytes ratio. Increased activation of $\mathrm{T}$ lymphocytes can become exhausting and show some features of inverted CD4/ $\mathrm{CD} 8$ ratio. Change in $\mathrm{T}$ lymphocytes affects the production of immunoglobulin gamma (IgG), leading to tissue damage. This condition could destroy the periodontium directly as manifestation. Also, SLE existence still has a late diagnosis, with ineffective treatments to cure both SLE and its complications such as periodontitis. ${ }^{12,13}$
Potential mechanisms into periodontitis start from dysregulation of the immune system; then there is a change in phagocyte activation after which inflammatory cytokines are induced, especially immunoglobulin gamma cytomegalovirus (IgG CMV), which contributes to tissue destruction in both SLE and periodontitis conditions. ${ }^{14,15}$ Immunological changes occurring through increasing $T$ cells, both CD4 and CD8, stimulate cytokines and create multiple organ destruction. Effector cells of the complement system also lead to tissue injury, which is a potential pathway for periodontitis in SLE. ${ }^{15,16}$ Role of CD4/CD8 ratio and IgG CMV in SLE patients with periodontitis in Indonesia has never been discussed before. Therefore, the aim of this study is to find the potential of CD4/CD8 ratio and IgG CMV in early diagnosis and in potential treatment of SLE subjects with periodontitis.

\section{Materials and Methods}

Research ethics was approved by Saiful Anwar Hospital Ethical Board (No. 400/120/K.3/302/2017). All samples were taken from Saiful Anwar Hospital, Rheumatology Department, Malang, Indonesia, and all subjects were required to sign informed consent showing willingness to be part of this study. The diagnosis of SLE was done by Rheumatologist in Internal Medicine Consultant. The research subjects were selected by purposive sampling technique based on criteria that had been set. Inclusion SLE criteria were women, age 18 to 55 years, and SLE diagnosed with excellent general condition. Exclusion SLE criteria were smoking habit and history of another systemic disease.

The design of the study was a cross-sectional approach with a total of 93 SLE subjects. Patient clinical assessment of SLE severity was done using Mexican SLE Disease Activity Index score, and oral cavities were assessed. Periodontitis was evaluated using the periodontal index (PI) by Periodontics. Then, SLE patients were analyzed for determining lymphocytes CD4/CD8 ratio using flow cytometry and IgG CMV levels using enzyme-linked immunosorbent assay (ELISA). PI assessment was done by a WHO Periodontal Examining Probe using Periodontal Index by Russel. This index uses the surface of the teeth. Scores 0 to 8 are assigned to each tooth, and the total score obtained from total findings is divided by all teeth in the oral cavity. ${ }^{17,18}$

\section{CD4/CD8 Ratio Using Flow Cytometry}

The sample used was peripheral blood mononuclear cell (PBMC), which was isolated from peripheral blood. Antibodies used are Biolegend FITC anti-human CD4 (300506) and Biolegend PerCp anti-human CD8 (344708). Further, $10^{3}$ to $10^{5}$ PBMC was added to specific primary antibodies with optimum concentration. The sample was incubated for 15 to 20 minutes in a dark room. After washing twice, to the sample was added secondary antibodies, namely fluorochrome conjugated anti-human immunoglobulin (FITC anti-human), and PerCP incubation was done on ice in a dark room for 15 to 20 minutes, then washed again. The pellet cells were resuspended in $0.5 \mathrm{~mL}$ cell-staining buffer and were 
Role of CD4/CD8 Ratio and IgG Cytomegalovirus as Potential Markers for SLE Patients with Periodontal

added $5 \mu \mathrm{L}(0.25 \mu \mathrm{g})$ viability-staining solution to remove the dead cells. Measurements were made at $10^{5}$ PBMC, and the results obtained in the form of a percentage (\%) of cells were analyzed using BD Cell Quest Pro software. ${ }^{19}$

\section{Levels of Immunoglobulin Gamma Cytomegalovirus} The levels of IgG CMV (Biovision ELISA Cat\#K4150-100) were determined using a sandwich ELISA commercial kit in $\mathrm{pg} / \mathrm{mL}$. Levels of IgG were measured using auto plate reader with optical density of $450 \mathrm{~nm} .^{20}$

\section{Statistical Analysis}

The differences among the three groups were analyzed using analysis of variance. Correlation among the groups was calculated using Spearman/Pearson correlation coefficient test, while regression analysis was done using Statistical Package for the Social Sciences, and the significant value found was $<0.05 .{ }^{21}$

\section{Results}

Overall, 93 SLE subjects were included in this study. As shown in - Fig. 1, the mean age of the SLE subject was 29; 58 subjects were below the age of 30 years while 35 subjects were over 30 years of age. Ethnically, 75 SLE patients were of Java ethnicity while 18 subjects belonged to other ethnicities. Among the SLE patients, 53 subjects were married while 40 were singles. Also, 25 were college graduates while 68 were school graduates. Employed subjects were 31 while 62 were unemployed.

Then, we evaluated the immune profile of the 93 SLE patients, such as CD4/CD8 ratio and levels of CMV IgG, and results can be seen in - Table 1. CD4/CD8 ratio was determined by flow cytometry method using PBMC whereas IgG CMV levels were determined by ELISA method using serum. Inverted CD4/CD8 ratio (<1) was found in SLE subjects with periodontitis, while eight SLE subjects were found with normal CD4/CD8 ratio (>1).
Table 1 Immune profile of SLE patients

\begin{tabular}{|l|l|l|l|}
\hline SLE $(\boldsymbol{n}=\mathbf{9 3})$ & Mean & SD & Range (Min-Max) \\
\hline CD4/CD8 ratio & 0.72 & 0.33 & $0.13-1.6$ \\
\hline IgG CMV & 1.66 & 0.77 & $0.08-3.22$ \\
\hline SLE severity & 17.70 & 12.70 & $0-32$ \\
\hline Periodontitis & 2.66 & 1.20 & $0.1-4.9$ \\
\hline
\end{tabular}

Abbreviations: IgG CMV, immunoglobulin gamma cytomegalovirus; Min-Max, minimum-maximum; SD, standard deviation; SLE, systemic lupus erythematosus.

Comparison of CD4/CD8 ratio between SLE subjects with and without periodontitis by flow cytometry method can be seen in - Fig. 2. SLE patients with periodontitis had inverted CD4/CD8 ratio. It can be seen that SLE patients with complications in periodontitis had higher CD8 than CD4 counts in percentage. SLE severity was found severely dominant; also, severe periodontitis was found to be dominant.

Comparison between CD4/CD8 ratio, IgG CMV levels, and periodontitis can be seen in - Table 2 based on SLE severity. In terms of SLE severity, mild, moderate, and severe groups showed significant differences with respect to CD4/CD8 ratio, IgG CMV levels, and periodontitis. CD4/CD8 ratio was found $<1$ or inverted dominant in severe activity. Moreover, higher IgG CMV levels and severe periodontitis were found in severe activity in SLE.

Correlation between CD4/CD8 ratio, IgG CMV levels, SLE activity, and periodontitis can be seen in - Table 3. Correlation between $\mathrm{CD} 4 / \mathrm{CD} 8$ ratio and periodontitis was found significant with a negative strong correlation $(r=-0.901)$, while correlation between IgG CMV levels and periodontitis was found significant with a positive strong correlation $(r=0.983)$. The graph of the correlation between markers and periodontitis can be seen in - Fig. 3. Moreover, correlation between CD4/CD8 ratio and SLE severity was found significant with a strong correlation $(r=-0.971)$. Also, IgG CMV levels and SLE severity were found significant with a strong correlation $(r=0.977)$.

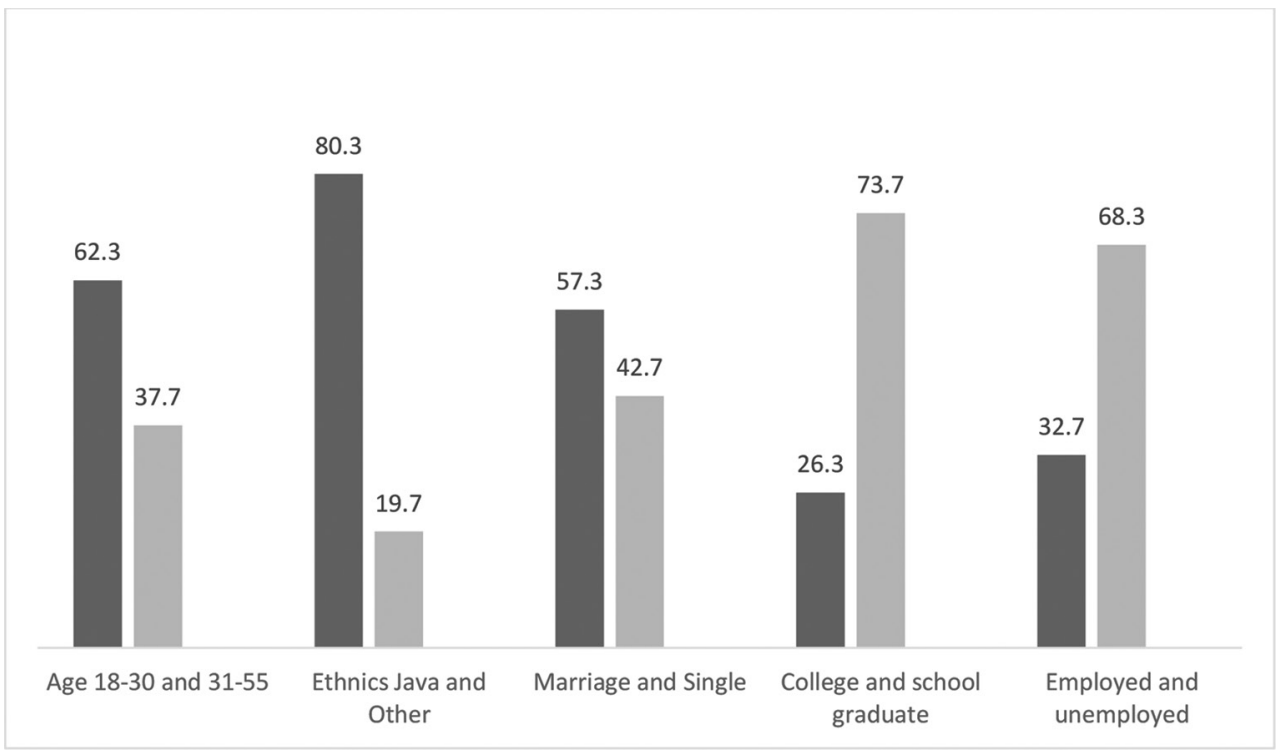

Fig. 1 Characteristics all systemic lupus erythematosus (SLE) patients in percentage. 


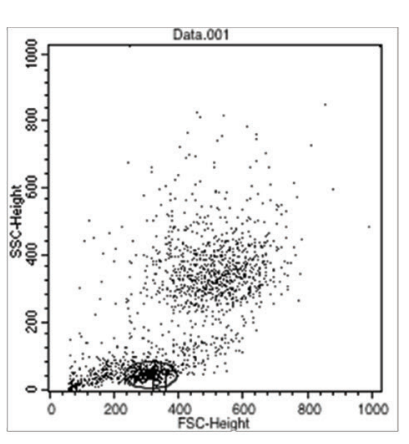

A
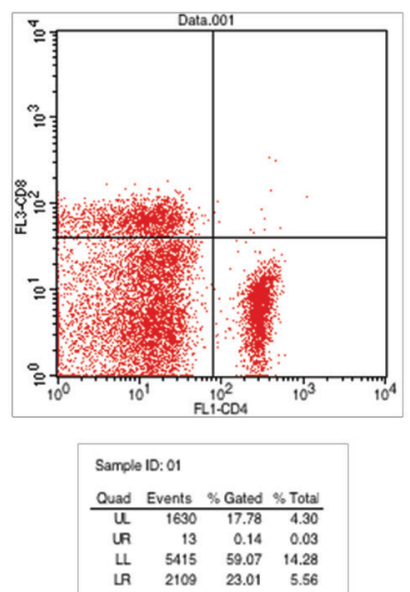

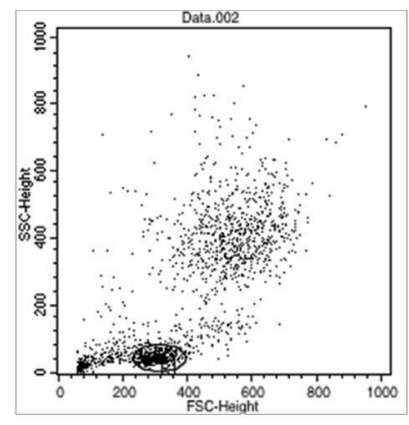

B
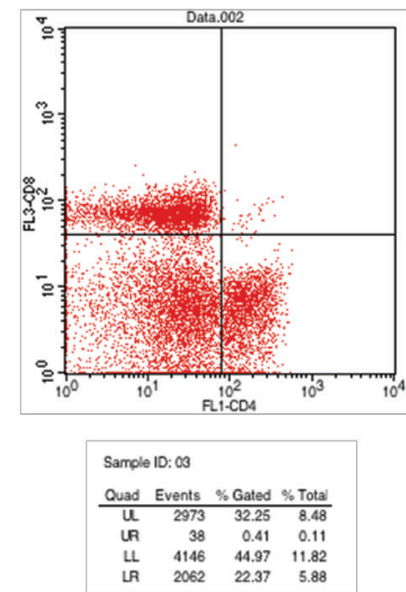

Fig. 2 Comparison of CD4/CD8 ratio in systemic lupus erythematosus patients (A) with and (B) without periodontitis using flow cytometry.

Table 2 Comparison of marker levels based on SLE severity

\begin{tabular}{|l|l|l|l|l|}
\hline Variables & $\begin{array}{l}\text { Mild } \\
(\boldsymbol{n}=32)\end{array}$ & $\begin{array}{l}\text { Moderate } \\
(\boldsymbol{n}=\mathbf{3 8})\end{array}$ & $\begin{array}{l}\text { Severe } \\
(\boldsymbol{n}=23)\end{array}$ & $p$-Value \\
\hline $\begin{array}{l}\text { CD4/CD8 } \\
\text { ratio (\%) }\end{array}$ & $1.19 \pm 0.24$ & $0.64 \pm 0.15$ & $0.26 \pm 0.08$ & $<0.001$ \\
\hline $\begin{array}{l}\text { IgG CMV } \\
(\mathrm{pg} / \mathrm{mL})\end{array}$ & $0.58 \pm 0.22$ & $1.63 \pm 0.45$ & $2.74 \pm 0.23$ & $<0.001$ \\
\hline $\mathrm{PI}$ & $0.75 \pm 0.59$ & $2.72 \pm 0.60$ & $4.20 \pm 0.33$ & $<0.001$ \\
\hline
\end{tabular}

Abbreviations: IgG CMV, immunoglobulin gamma cytomegalovirus; PI, periodontal index; SLE, systemic lupus erythematosus.

Table 3 Correlation between marker levels, SLE severity, and periodontitis

\begin{tabular}{|c|c|c|}
\hline Variables & $\begin{array}{l}p \text {-Value } \\
\text { (Sig, 2-tailed) }\end{array}$ & $r$ \\
\hline \multicolumn{3}{|l|}{ Marker levels and periodontitis } \\
\hline $\begin{array}{l}\text { CD4/CD8 ratio and } \\
\text { periodontitis }\end{array}$ & $<0.001$ & -0.901 \\
\hline IgG CMV and periodontitis & $<0.001$ & 0.983 \\
\hline \multicolumn{3}{|l|}{ Marker levels and SLE severity } \\
\hline CD4/CD8 ratio and SLE & $<0.001$ & -0.971 \\
\hline $\operatorname{lgG}$ CMV (pg/mL) and SLE & $<0.001$ & 0.977 \\
\hline
\end{tabular}

Abbreviations: IgG CMV, immunoglobulin gamma cytomegalovirus; SLE, systemic lupus erythematosus.

- Table 4 provides a regression analysis of marker levels (CD4/CD8 and IgG CMV), SLE severity, and periodontitis. It explains that marker levels such as CD4/CD8 ratio and $\operatorname{IgG}$ CMV have a direct and moderate impact on periodontitis and SLE severity. Furthermore, path analysis is needed to find indirect pathway markers and periodontitis.

Receiver operating curve of CD4/CD8 ratio and IgG CMV can be seen in - Fig. 4. CD4/CD8 ratio was found significant $(p=<0.001)$ with a wide area of sensitivity and specificity (0.869). IgG CMV was found significant $(p=<0.001)$ with a wide area of sensitivity and specificity (0.852).

\section{Discussion}

Periodontitis is a chronic disease involving destruction of periodontal tissue. Periodontal damage results in gum bleeding and attachment loss, and finally loss of tooth. Periodontitis is initiated by a local accumulation of bacteria and sometimes worsens by systemic disease. In this case, SLE could increase the severity of periodontal diseases. The host response condition in periodontitis is influenced by inflammation responses from the immune system. .,12 $^{3}$

Our results showed significant differences between SLE and healthy subjects with periodontitis. Previous studies have also reported that SLE has two times higher periodontitis incidence than healthy subjects, in countries such as the UK, the USA, and Japan. SLE subjects had tendencies of having periodontitis due to autoimmune conditions. Hyperreactivity of immune response could induce lymphocytes, resulting in high inflammation. ${ }^{22,23}$ There was a study about SLE that showed no impact on periodontal disease, but this condition may be due to small sample size $(n=<20)$, treatment factor, and other systemic diseases. ${ }^{24}$ Between the two groups, SLE severity was found having a significant difference in periodontitis. Higher disease activity in SLE, maybe, had a significant role in periodontitis severity. ${ }^{25}$

Studies have suggested variations in response of the immune system in SLE patients. Polymorphism of cytokine markers was related to periodontitis. Fc receptor and IgG also had important roles in the regulation. Cytokine receptors and antibodies stimulate bone resorption by directly inducing proliferation of osteoclast progenitors and also inducing osteoclast maturation. ${ }^{25,26}$ Prevalent theories concerning SLE patients believe that cytokines and antibodies are correlated with disease activity, and are also associated with a possible role in periodontitis. Cytokine serum levels were changing in adolescents with SLE compared with healthy subjects and correlated positively with the SLE activity index. ${ }^{27}$

Another potential mechanism is the presence of changes in endothelial cells. IgG CMV levels are elevated in patients with SLE. In fact, infection could be regarded as a triggering factor that results in autoimmune diseases. It has been reported that SLE in children and adults is triggered due to a secondary 

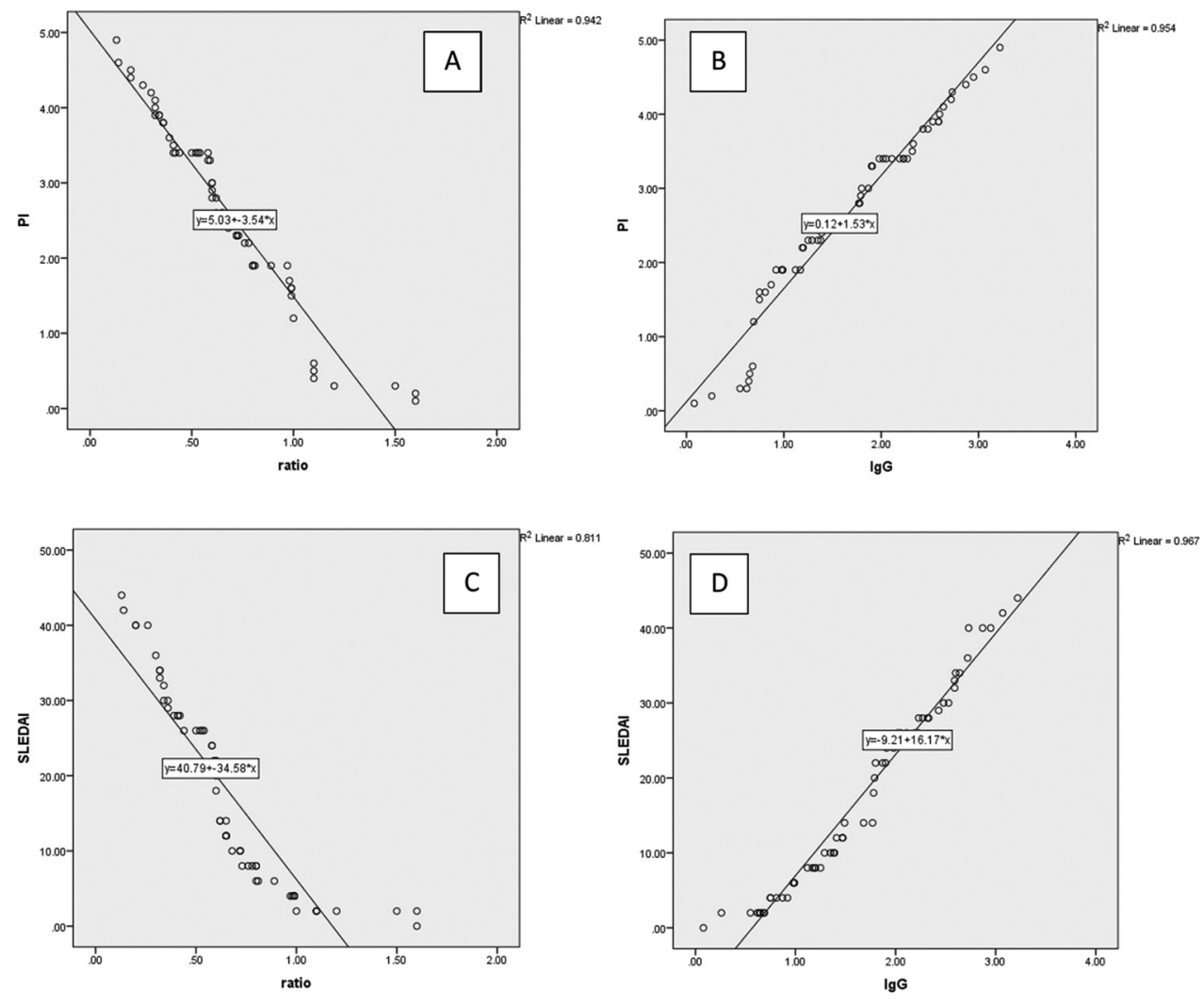

Fig. 3 (A) Correlation between CD4/CD8 ratio and periodontitis and (B) between immunoglobulin gamma cytomegalovirus (IgG CMV) levels and periodontitis in systemic lupus erythematosus (SLE) patients. (C) Correlation between CD4/CD8 ratio and SLE severity and (D) between IgG CMV levels and SLE severity in SLE patients. PI, periodontal index.

Table 4 Regression between marker levels, SLE, and periodontitis

\begin{tabular}{|c|c|c|}
\hline Variables & $\begin{array}{l}p \text {-Value } \\
\text { (Sig, 2-tailed) }\end{array}$ & $\begin{array}{l}\text { Coefficient } \\
\beta\end{array}$ \\
\hline \multicolumn{3}{|l|}{ Marker levels and periodontitis } \\
\hline $\begin{array}{l}\text { CD4/CD8 ratio and } \\
\text { periodontitis }\end{array}$ & $<0.001$ & -0.434 \\
\hline $\operatorname{lgG}$ CMV and periodontitis & $<0.001$ & 0.562 \\
\hline \multicolumn{3}{|l|}{ Marker levels and SLE } \\
\hline CD4/CD8 ratio and SLE & $<0.001$ & -0.435 \\
\hline $\operatorname{lgG}$ CMV (pg/mL) and SLE & $<0.001$ & 1.380 \\
\hline
\end{tabular}

Abbreviations: IgG CMV, immunoglobulin gamma cytomegalovirus; SLE, systemic lupus erythematosus.

stimulus such as an infection. Infection could induce abnormal cytokine and immune responses, and explain higher IgG CMV in SLE periodontitis as interplay. ${ }^{28,29}$

Recent literature is available regarding lymphocytes analysis associated with an inverted CD4/CD8 ratio less than 1 . Normally, CD4/CD8 ratio is higher than 1 in physiologic conditions. Our study on SLE patients also found that CD4/ CD8 ratio $<1$ was associated with a higher prevalence of periodontitis. Repeated and persistent stimulation of antigens in SLE patients leads to increasing $\mathrm{T}$ cell expressions in CD8+ and decreasing in CD4+. This condition resulting in SLE patients is more susceptible to activated cell-induced cell death, stimulated by mitogen and high risk of infection. The evidence suggests that cell can be used also as a marker for periodontitis in SLE condition. ${ }^{30-32}$

On the other hand, a lot of investigations have been conducted to identify the specific role of lymphocytes B, especially immunoglobulins associated with risk for periodontal diseases. One marker for SLE is higher IgG CMV levels due to higher risk of infection..$^{29}$ Our study also found higher IgG CMV levels, and other systemic conditions such as human immunodeficiency virus (HIV) infection and rheumatoid arthritis. ${ }^{25,30}$ It is believed that IgG CMV levels are hallmarks of apoptosis and tissue damage also. In a previous study on HIV patients with periodontitis, expression of IgG CMV was shown to increase in many cases. Another study found IgG associated with bacterial periodontitis directly, resulting in a higher prevalence of periodontitis. ${ }^{29,31}$ This study also found that IgG CMV levels played a critical role in the regulation of host immune response and inflammation. This pathway can be modulated by various stimuli, including cytokines and immune cells themselves. ${ }^{27}$

SLE may increase inflammatory response in periodontal disease through immune response activation by lymphocytes $\mathrm{T}$ and $\mathrm{B}$. Lymphocytes are activated by innate immunity that recognizes invasion of pathogenic microorganisms and initiates signal transduction. Signal pathways are regulated by immunoglobulins, especially IgG. ${ }^{30,32}$ Furthermore, CD4/CD8 ratio and IgG CMV could be helpful in early diagnosis of SLE 

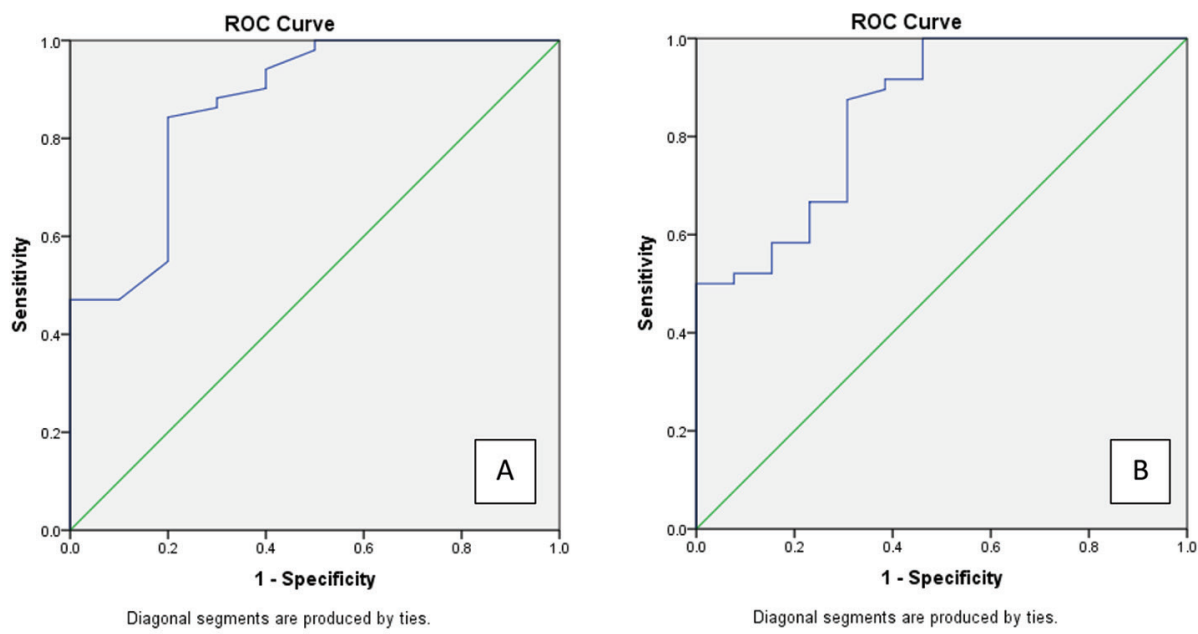

Diagonal segments are produced by ties.

Fig. 4 Receiver operating curve (ROC) of CD4/CD8 ratio and immunoglobulin gamma cytomegalovirus.

and as potential treatment for SLE severity and its complications such as periodontitis. This treatment should be responsible for the maintenance of SLE severity. Further studies are required for confirming lymphocytes and IgG CMV as potential options for treatment, taking into consideration toxicity and effectivity tests.

\section{Conclusions}

Periodontal disease prevalence was found to be higher, along with more severe SLE. Inverted CD4/CD8 ratio and IgG CMV were also found to be associated with SLE patients with periodontitis. It is recommended that further studies are needed to confirm CD4/CD8 and IgG CMV as potential markers for treatment of SLE with periodontitis.

\section{Conflict of Interest}

None declared.

\section{References}

1 Fabbri C, Fuller R, Bonfá E, Guedes LK, D’Alleva PS, Borba EF. Periodontitis treatment improves systemic lupus erythematosus response to immunosuppressive therapy. Clin Rheumatol 2014;33(4):505-509

2 Petri M, Orbai AM, Alarcón GS, et al. Derivation and validation of the Systemic Lupus International Collaborating Clinics classification criteria for systemic lupus erythematosus. Arthritis Rheum 2012;64(8):2677-2686

3 Calderaro DC, Ferreira GA, Corrêa JD, et al. Is chronic periodontitis premature in systemic lupus erythematosus patients? Clin Rheumatol 2017;36(3):713-718

4 Rutter-Locher Z, Fuggle N, Orlandi M, D'Aiuto F, Sofat N. Periodontal disease and autoimmunity: what we have learned from microbiome in rheumatology. J Intech 2017;6:127-129

5 Kobayashi T, Ito S, Yamamoto K, et al. Risk of periodontitis in systemic lupus erythematosus is associated with Fcgamma receptor polymorphisms. J Periodontol 2003;74(3):378-384

6 Gofur NRP, Nurdiana N, Handono K, Kalim H. Periodontal tissue condition on systemic lupus erythematosus patients: a clinical study. Pesqui Bras Odontopediatria Clin Integr 2020;20(1):5094-5102

7 Gofur NRP, Nurdiana N, Kalim H, Handono K. Periodontitis is associated with disease severity and anti-double stranded DNA antibody and interferon-gamma levels in patients with systemic lupus erythematosus. J Taibah Univ Med Sci 2019;14(6):560-565

8 Gofur NRP, Nurdiana N, Handono K, Kalim H. Immune aging marker associated with periodontitis in systemic lupus erythematosus patients. AIP Conf Proc 2019;5(1):1-11

9 Cullinan MP, Seymour GJ. Periodontal disease and systemic illness: will the evidence ever be enough? Periodontol 2000 2013;62(1):271-286

10 Genco RJ, Borgnakke WS. Risk factors for periodontal disease. Periodontol 2000 2013;62(1):59-94

11 Bartold PM, Van Dyke TE. Periodontitis: a host-mediated disruption of microbial homeostasis. Unlearning learned concepts. Periodontol 2000 2013;62(1):203-217

12 Ebersole JL, Dawson III DR, Morford LA, Peyyala R, Miller CS, Gonzaléz OA. Periodontal disease immunology: 'double indemnity' in protecting the host. J Periodontol 2000;2013(62):163-202

13 Marques CP, Maor Y, de Andrade MS, Rodrigues VP, Benatti BB. Possible evidence of systemic lupus erythematosus and periodontal disease association mediated by toll-like receptors 2 and 4. Clin Exp Immunol 2016;183(2):187-192

14 Kaur G, Mohindra K, Singla S. Autoimmunity - basics and link with periodontal disease. Autoimmun Rev 2017;16(1):64-71

15 Megson E, Fitzsimmons T, Dharmapatni K, Bartold PM. C-reactive protein in gingival crevicular fluid may be indicative of systemic inflammation. J Clin Periodontol 2010;37(9):797-804

16 Sete MRC, Figueredo CMS, Sztajnbok F. Periodontitis and systemic lupus erythematosus. Rev Bras Reumatol Engl Ed 2016;56(2):165-170

17 Beltrán-Aguilar ED, Eke PI, Thornton-Evans G, Petersen PE. Recording and surveillance systems for periodontal diseases. Periodontol 2000 2012;60(1):40-53

18 Rebelo MAB, de Queiroz AC, Gingival indices: state of art. In: Panagakos E, ed. Gingival Diseases. Their Aetiology, Prevention and Treatment. London: IntechOpen; 2011:41:54, ; chap. 3

19 Ormerod MG. Flow cytometry: a basic introduction. De Novo Journal 2008;4(1):73-85 
20 Gan SD, Patel KR. Enzyme immunoassay and enzyme-linked immunosorbent assay. J Invest Dermatol 2013;133(9):e12

21 Mukaka MM. Statistics corner: a guide to appropriate use of correlation coefficient in medical research. Malawi Med J 2012;24(3):69-71

22 Rees F, Doherty M, Grainge MJ, Lanyon P, Zhang W. The worldwide incidence and prevalence of systemic lupus erythematosus: a systematic review of epidemiological studies. Rheumatology (Oxford) 2017;56(11):1945-1961

$23 \mathrm{Wu}$ YD, Lin CH, Chao WC, Liao TL, Chen DY, Chen HH. Association between a history of periodontitis and the risk of systemic lupus erythematosus in Taiwan: a nationwide, population-based, case-control study. PLoS One 2017;12(10):e0187075

24 Al-Mutairi KD, Al-Zahrani MS, Bahlas SM, Kayal RA, Zawawi KH. Periodontal findings in systemic lupus erythematosus patients and healthy controls. Saudi Med J 2015;36(4):463-468

25 Albandar JM, Susin C, Hughes FJ. Manifestations of systemic diseases and conditions that affect the periodontal attachment apparatus: case definitions and diagnostic considerations. J Periodontol 2018;89(Suppl 1) :S183-S203

26 Hammoudeh M, Al-Momani A, Sarakbi H, Chandra P, Hammoudeh S. Oral manifestations of systemic lupus erythematosus patients in Qatar: a pilot study. Int J Rheumatol 2018;2018:6052326

27 Nair S, Faizuddin M, Dharmapalan J. Role of autoimmune responses in periodontal disease. Autoimmune Dis 2014;2014:596824

28 Kaur G, Grover V, Bhaskar N, Kaur RK, Jain A. Periodontal infectogenomics. Inflamm Regen 2018;38:8-25

29 Damgaard C, Reinholdt J, Enevold C, Fiehn NE, Nielsen $\mathrm{CH}$, Holmstrup P. Immunoglobulin $\mathrm{G}$ antibodies against Porphyromonas gingivalisorAggregatibacter actinomycetemcomitansin cardiovascular disease and periodontitis. J Oral Microbiol 2017;9(1):1374154

30 Lee SA, Sinclair E, Hatano H, et al. Impact of HIV on CD8+ T cell CD57 expression is distinct from that of CMV and aging. PLoS One 2014;9(2):e89444

31 Fonseca RR, Alves ACB, Amanajás TA, et al. Absence of cytomegalovirus in gingivitis and chronic periodontitis in HIV-1 patients in Northern Brazil. Rev Soc Bras Med Trop 2018;51(6):809-812

32 Manjarrez-orduño N, Menard LC, Carman JA. A systemic lupus erythematosus endophenotype characterized by increased CD8 cytotoxic signature associates with renal involvement. ImmunoHorizons 2017;1(7):124-132 